\title{
Abnormal spectral evolution of fiber Bragg gratings in hydrogenated fibers
}

\author{
Y. Liu, J. A. R. Williams, L. Zhang, and I. Bennion \\ Photonics Research Group, School of Engineering and Applied Science, Aston University, Aston Triangle, \\ Birmingham B4 7ET, UK
}

Received October 25, 2001

\begin{abstract}
We report the observation of abnormal spectral evolution in regenerated fiber Bragg gratings in hydrogenated B-Ge-codoped and standard telecom fiber with UV overexposure. The behavior of this new type of regenerated grating, which we have designated type IA, contrasts with that of the previously reported type IIA grating in nonhydrogenated fiber by exhibiting a large redshift in Bragg wavelength with increasing exposure of as much as $18 \mathrm{~nm}$ from a strong (16-dB) regenerated grating in B-Ge fiber. (C) 2002 Optical Society of America
\end{abstract}

OCIS codes: $\quad 060.2400,060.2310,060.2270$.

Exposing the core of a photosensitive optical fiber to a UV laser interference pattern changes the fiber's refractive index periodically, producing a fiber Bragg grating (FBG). ${ }^{1} \quad$ A number of FBG types have been distinguished, characterized by markedly different spectral and thermal behaviors, that originate from the UV excitation of several distinct physical mechanisms. During the typical inscription process, the central wavelength of the grating increases with the UV exposure, which is indicative of the induction of a positive change in refractive index. The resultant FBG has come to be referred to as a type I grating and is the type most commonly fabricated by either a free-space holographic or a phase-mask exposure technique; however, this FBG is the least stable at high temperatures of the type reported to date. With further UV exposure the type I grating growth saturates, and formation of the so-called type IIA grating commences. ${ }^{2}$ The observed reflectivity decreases almost to zero before increasing again and, eventually, saturating; the central refractive wavelength decreases during this stage of the exposure, indicating a negative induced index change. Type IIA gratings are usually inscribed at a wavelength of $193 \mathrm{~nm},{ }^{2-4}$ and, although the underlying mechanism is still not wholly clarified, links to stress and defect concentrations have been identified. ${ }^{5-7}$

In all observations reported to date, the type IIA gratings were formed in nonhydrogenated fibers. However, the introduction of hydrogen into the fiber to increase photosensitivity is a commonly adopted procedure. In seeking to clarify FBG growth with UV overexposure in hydrogenated standard telecom and B-Ge-codoped fibers we have observed, for the first time to our knowledge, regenerated grating growth with an abnormal, i.e., contrary to the behavior expected on the basis of previously reported observation, spectral evolution that is quite distinct from that of type IIA. We have designated this new grating type IA and found it to exhibit less temperature sensitivity than the type I grating.

0146-9592/02/080586-03\$15.00/0
We inscribed the FBGs reported here by two-beam holographic exposure, using a cw 244-nm laser with $8-\mathrm{mm}$ beam width and $60-\mathrm{mW}$ power. For reference, we produced a type I FBG in nonhydrogenated B-Ge-codoped fiber: a maximum 2.2- $\mathrm{dB}$ reflectivity was obtained in $\sim 2$ min of UV exposure, as shown in Fig. 1(a); the reflectivity decreased with subsequent exposure and fell to zero after $7 \mathrm{~min}$. A maximum redshift of $\sim 2.0 \mathrm{~nm}$ in the Bragg wavelength was recorded. As the UV exposure was maintained, the grating reflectivity increased from zero, accompanied by a blueshift in the Bragg wavelength that is characteristic of the type IIA grating and a negative induced refractive-index change. Limited by the UV wavelength and power, the type IIA grating attained 8.7- $\mathrm{dB}$ reflectivity and 0.66-nm blueshift after 37-min exposure. Subsequent annealing of the grating for $12 \mathrm{~h}$ at $70^{\circ} \mathrm{C}$ induced a further blueshift of $0.55 \mathrm{~nm}$ owing to relaxation of the photoinduced positive index change; the reflectivity remained unchanged, indicating good thermal stability, as shown in Fig. 1(b).

This fabrication procedure was repeated with a hydrogenated sample of the same fiber. Type I grating formation and complete erasure were again observed; however, with continued UV exposure a quite different behavior emerged that included formation of an

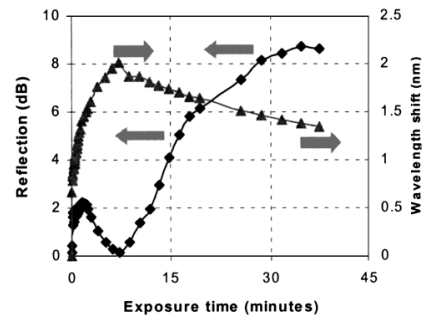

(a)

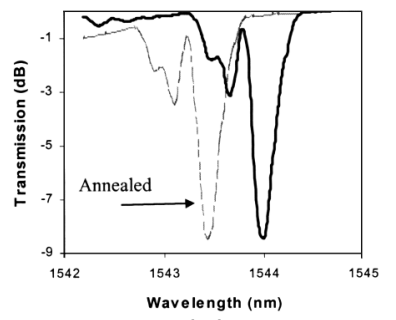

(b)
Fig. 1. Formation of an 8-mm-long type IIA grating in nonhydrogenated B-Ge-codoped fiber: (a) reflectivity and wavelength shift relative to exposure time, (b) transmission spectra before and after annealing. 
(a)

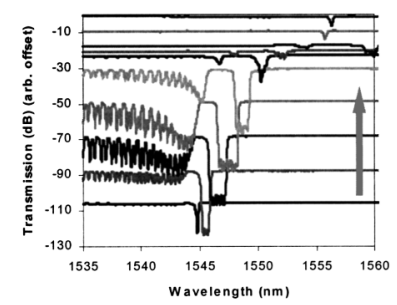

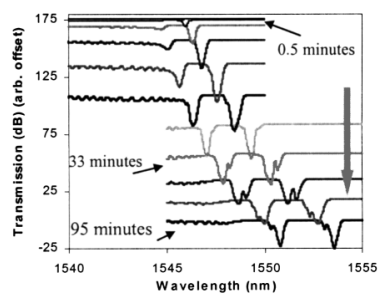

(b)
Fig. 2. Transmission spectra for 8-mm-long gratings inscribed in hydrogenated fibers, illustrating abnormal spectral evolution with increasing UV exposure (the direction of the large, bold arrow): (a) in B-Ge-codoped fiber and (b) in standard fiber.

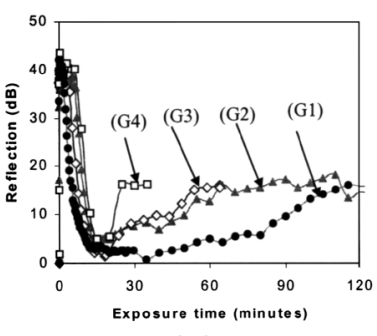

(a)

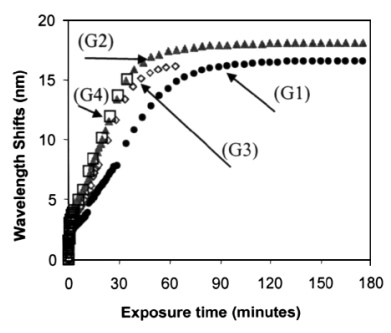

(b)
Fig. 3. Formation of four 8-mm-long gratings (G1)-(G4) in hydrogenated B-Ge-codoped fiber: (a) reflectivity and (b) Bragg wavelength shift, plotted relative to exposure time.

abnormal grating spectrum and a significant redshift, as illustrated by Fig. 2(a). Similar behavior was obtained with hydrogenated standard fiber, as shown in Fig. 2(b). In Fig. 3 we have plotted the measured reflectivities and wavelength shifts relative to UV exposure time for a series of four gratings, (G)-(G4), inscribed in the hydrogenated $\mathrm{B}-\mathrm{Ge}$ fiber. The behavior is similar in all cases: After $\sim 15$ min the type I gratings are completely erased, and after $\sim 60 \mathrm{~min}$ the new spectra have formed and the regenerated gratings (type IA) have attained their maximum reflectivity, $\sim 16 \mathrm{~dB}$, with corresponding redshifts as high as $18 \mathrm{~nm}$. With longer exposure (we continued for 4 and $6.5 \mathrm{~h}$ ), no significant further changes in either the reflectivity or the wavelength shift were observed. For this fiber, we calculate that the 18-nm redshift corresponds to a positive refractive-index change of $\Delta n=1.68 \times 10^{-2}$.

It is noteworthy that the calculated value of $\Delta n$ for the exposed fiber core yields an increase in the numerical aperture of the fiber by a factor 2.6. In Fig. 4 a higher-order core mode is apparent, with 6 - $\mathrm{dB}$ reflectivity, separated from the primary Bragg resonance by $5.9 \mathrm{~nm}$. (Higher-order modes are also apparent in Fig. 2.) After annealing for $12 \mathrm{~h}$ at $70{ }^{\circ} \mathrm{C}$, the regenerated grating exhibited a Bragg wavelength shift to a shorter wavelength of $2-3 \mathrm{~nm}$ and a significant decrease in coupling strength, also shown in Fig. 4.

Figure 4 also shows the overall reflection spectrum of the regenerated grating. Two plateaus are apparent; the first, commencing at $\lambda_{1} \sim 1545 \mathrm{~nm}$, corresponds to the remnant type I region, and the second, commencing at $\lambda_{2} \sim 1550 \mathrm{~nm}$, is indicative of the type IA region. The higher-order core mode is clearly visible in the spectrum at $\lambda_{3}$.

Finally, we investigated the temperature sensitivity of the type IA grating compared with the initial type I grating by measuring the Bragg wavelength shifts as the sample temperature was raised; the results for one sample are shown in Fig. 5. Whereas the type I grating shows a Bragg wavelength shift of $8.9 \mathrm{pm} / \mathrm{K}$, the regenerated grating exhibits the somewhat lower sensitivity of $6.4 \mathrm{pm} / \mathrm{K}$.

In conclusion, the properties of fiber Bragg gratings under conditions of UV overexposure in B-Ge-codoped and standard fibers have been investigated experimentally. In nonhydrogenated fiber, thermally stable type IIA gratings have been observed. In hydrogenated fiber, significantly different behavior was observed, with abnormal spectral evolution of regenerated grating following erasure of the initial type I grating. A substantial shift of the Bragg wavelength by as much as $18 \mathrm{~nm}$ to a longer wavelength, implying a refractive-index increase as large as $1.68 \times 10^{-2}$, is in marked contrast to the shift to shorter wavelength and the negative index change of the type IIA grating. The regenerated grating also exhibits less temperature sensitivity than the type I grating.

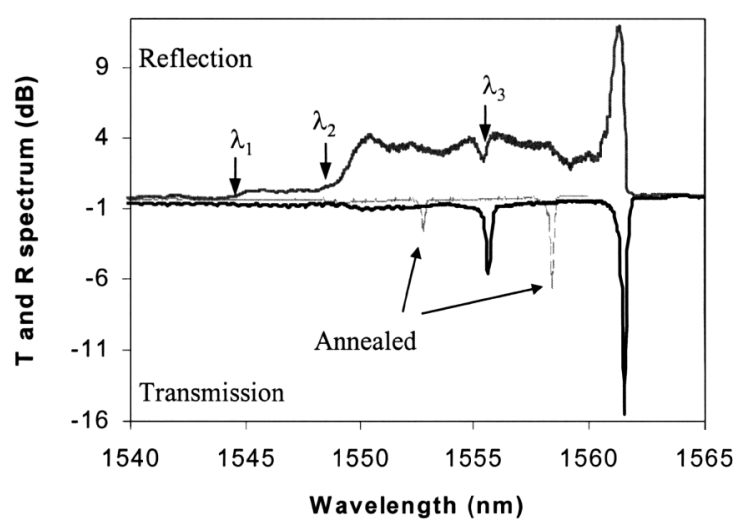

Fig. 4. Reflection (R) and transmission (T) spectra of a regenerated grating after $\sim 1$-h exposure in hydrogenated B-Ge-codoped fiber.

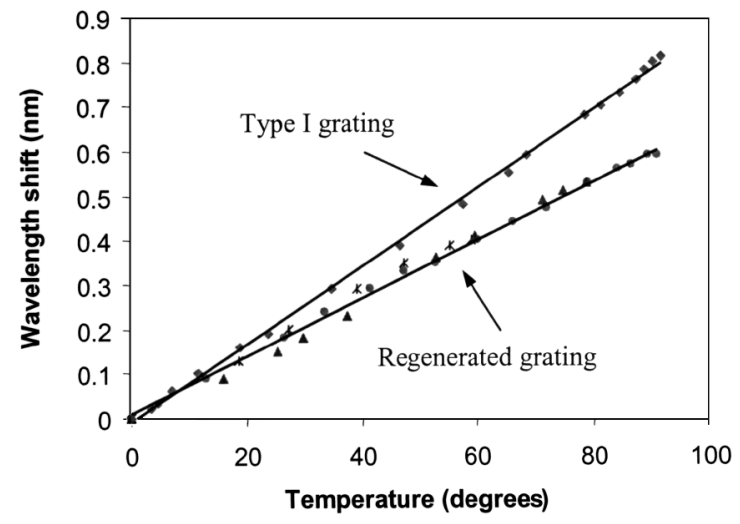

Fig. 5. Bragg wavelength shifts measured in type I and regenerated (type IA) gratings inscribed in hydrogenated B-Ge fiber plotted versus temperature. Symbols, experimental points; solid curves, best fits to the data. 
This research was carried out with the support of the UK Engineering and Physical Sciences Research Council. We acknowledge the provision of B-Ge-codoped fiber by Nortel, UK. Y. Liu's e-mail address is liuy@aston.ac.uk.

\section{References}

1. R. Kashyap, Fibre Bragg Gratings (Academic, London, 1999).

2. I. Riant and F. Haller, J. Lightwave Technol. 15, 1464 (1997).

3. W. X. Xie, P. Niay, P. Bernage, M. Douay, J. F. Bayon, T. Georges, M. Monerie, and B. Poumellec, Opt. Commun. 104, 185 (1993).
4. M. Douay, W. X. Xie, T. Taunay, P. Bernage, P. Niay, P. Cordier, B. Poumellec, L. Dong, J. F. Bayon, H. Poignant, and E. Delevaque, J. Lightwave Technol. 15, 1329 (1997).

5. J. Canning, D. Moss, M. Aslund, and M. Bazylenko, Electron. Lett. 34, 366 (1998).

6. L. Dong, W. F. Liu, and L. Reekie, Opt. Lett. 21, 2032 (1997).

7. W. X. Xie, P. Niay, P. Bernage, M. Douay, T. Taunay, J. F. Bayon, E. Delevaque, and M. Monerie, Opt. Commun. 124, 295 (1996). 